\title{
Markers of Increased Cardiovascular Risk in Postmenopausal Women: Focus on Oxidized-LDL and HDL Subpopulations
}

\author{
Filipa Mascarenhas-Melo, ${ }^{1}$ José Sereno, ${ }^{1}$ Edite Teixeira-Lemos, ${ }^{1,2}$ Sandra Ribeiro, ${ }^{3,4}$ \\ Petronila Rocha-Pereira, ${ }^{4,5}$ Ethan Cotterill, ${ }^{6}$ Frederico Teixeira, ${ }^{1}$ and Flávio Reis ${ }^{1}$ \\ ${ }^{1}$ Laboratory of Pharmacology \& Experimental Therapeutics, IBILI, Faculty of Medicine, University of Coimbra, \\ 3000-548 Coimbra, Portugal \\ ${ }^{2}$ ESAV and Educational Technologies and Health Study Centre, Polytechnic Institute of Viseu, 3504-510 Viseu, Portugal \\ ${ }^{3}$ Service of Biochemistry, Faculty of Pharmacy, University of Porto, 4050-313 Porto, Portugal \\ ${ }^{4}$ Institute of Molecular and Cellular Biology, University of Porto, 4150-180 Porto, Portugal \\ ${ }^{5}$ Research Centre for Health Sciences, Beira Interior University, 6201-001 Covilhã, Portugal \\ ${ }^{6}$ Centre for Mathematics, University of Coimbra, 3001-454 Coimbra, Portugal
}

Correspondence should be addressed to Flávio Reis; freis@fmed.uc.pt

Received 14 February 2013; Accepted 8 May 2013

Academic Editor: Sudhir Srivastava

Copyright (C) 2013 Filipa Mascarenhas-Melo et al. This is an open access article distributed under the Creative Commons Attribution License, which permits unrestricted use, distribution, and reproduction in any medium, provided the original work is properly cited.

\begin{abstract}
Objective. To evaluate the effect of gender and menopause in cardiovascular risk (CVR) in a healthy population based on both classical and nontraditional markers. Methods. 56 men and 68 women (48 pre- and 20 postmenopause) were enrolled in the study. The following markers were analyzed: blood pressure (BP), body mass index (BMI), waist circumference (WC), glucose, total cholesterol (total-c), triglycerides (TGs), low-density lipoprotein cholesterol (LDL-c), oxidized-LDL (Ox-LDL), HDL-c and subpopulations, paraoxonase-1 activity, hsCRP, uric acid, tumor necrosis factor alpha (TNF- $\alpha$ ), adiponectin, vascular endothelial growth factor (VEGF), and intercellular adhesion molecular 1 (ICAM1). Results. Relative to the women, men present significantly increased BMI, WC, BP, glucose, total-c, TGs, LDL-c, Ox-LDL, uric acid, and TNF- $\alpha$ and reduced adiponectin and total and large HDL-c. The protective profile of women is lost after menopause with a significantly increased BMI, WC, BP, glucose, LDL-c, OxLDL, hsCRP, and VEGF and decreased total and large HDL-c. Significant correlations were found in women population and in postmenopausal women between Ox-LDL and total, large, and small HDL-c and between TNF- $\alpha$ and total, large, and small HDLc, LDL-c, and Ox-LDL. Conclusions. Men present higher CVR than women who lost protection after menopause, evidenced by nontraditional markers, including Ox-LDL and HDL subpopulations.
\end{abstract}

\section{Introduction}

Cardiovascular disease (CVD) is the leading cause of morbidity and mortality in men and women worldwide [1]. The apparent cardioprotective effects of endogenous estrogens seem to prevent CVD in premenopausal women, when compared with age-matched men; following menopause and the consequent loss of hormonal effects, gender-based differences in CVD are reduced [1], but the precise causes remain to be fully elucidated. Although previous studies have spotlighted the effects of estrogens, no conclusive evidence has proven their role in reducing the incidence of CVD
[2], and some studies indicate that the cardiovascular effects usually attributed to menopause are merely a consequence of the older age of menopausal women $[3,4]$. So it seems clear that more studies are needed to understand the precise influence of gender and menopause in cardiovascular risk (CVR). Moreover, it seems imperative to develop a more flexible technique, using not only the classical markers of CVD but also new "nontraditional" ones that have been increasingly associated with disease and pathology.

Chronic inflammation is currently viewed as a key factor in the development of atherosclerosis, contributing to raise the overall CVR in CVD populations. An inflammatory 
imbalance, as manifested by increased proinflammatory cytokines, such as the tumor necrosis factor alpha (TNF- $\alpha$ ) and/or reduced levels of anti-inflammatory and antiatherogenic mediators such as adiponectin, has been considered a key factor for the increased CVR in some pathology $[5,6]$ and thus deserves more attention in the menopausal stage. Similar importance is now attributed to the phenomenon of angiogenesis, which has the vascular endothelial growth factor (VEGF) as a (new and increasingly important) biomarker [7].

Low-density lipoprotein (LDL) oxidation is associated with coronary artery disease (CAD), since it is a promoter of key steps in the onset and evolution of atherosclerosis, including stimulation of monocyte infiltration and smooth muscle cell migration and proliferation; conversely, high levels of high-density lipoprotein cholesterol (HDL-c) prevents the development of atherosclerosis and CAD, in particular due to the transport of reserve cholesterol and the inhibition of oxidized LDL- (Ox-LDL-) induced monocyte infiltration [8]. The functionality, in addition to the concentration, of lipoproteins seems to play a role in the development and progression of atherosclerosis. Paraoxonase 1 (PON1) is an HDL-containing enzyme with antiatherogenic and antioxidant properties, including protection against LDL oxidation [9]. In fact, Ox-LDL and HDL are indeed antagonists in the development of CVD [7]. Several studies have shown a strong negative correlation between their levels and the development of atherosclerosis, but their role in the determination of CVR in gender and menopause remains to be clarified $[10,11]$. Recent findings suggest that monitoring the type of HDL particles (carry distinct and specific proteins or lipids and differentiated by their density and size-large, intermediate, and small), rather than their total quantity, is a more reasonable way of determining the $\mathrm{CV}$ risk, suggesting that different subpopulations may have a different role in reverse cholesterol transport and CVD risk protection [12]. In fact, some recent studies have been reporting that large HDL levels are reduced in patients with CAD compared to healthy subjects and inversely related to both disease severity and progression of coronary lesions [13].

This study aimed to evaluate the influence of gender and menopause on CVR in a population of healthy volunteers, using both traditional and new nontraditional markers and focusing on Ox-LDL and on HDL subpopulations given their recognized association with CVD.

\section{Materials and Methods}

2.1. Subjects and Ethical Consideration. One hundred and twenty-four volunteers, including 56 men and 68 women (48 pre- and 20 postmenopausal), aged from 16 to 75 , were randomly recruited during the performance of routine laboratory analysis in a clinical laboratory. The volunteers were selected after not expressing any diagnosis or taking medication for CVD, no family history of cardiovascular disease, and with serum levels of traditional routine lipid measures and glycemia within the normal range. Written informed consent was obtained from volunteers, each of whom completed the same questionnaire. Participants were not using exogenous steroids, not taking any medication, and did not declare any disease. Menopausal status (pre- and postmenopausal women) was defined by the questionnaire, and menstrual status was self-reported during the interviews. The definition of the World Health Organization [14] was used, which considers postmenopausal status as absence of menstruation for at least 12 months. The mean duration of menopause in our sample was $11.9 \pm 1.6$ years. No surgical-evoked menopause was included. Pregnant women were excluded from this study. The smoking habits of the populations were self-reported as (a) men: 47 nonsmokers and 9 smokers; (b) women: 56 nonsmokers and 12 smokers; (c) premenopausal women: 37 nonsmokers and 11 smokers; (d) postmenopausal women: 19 non-smokers and 1 smoker. The study was performed in agreement with the code of ethics of the World Medical Association (Declaration of Helsinki) and received authorization from the local ethics committee.

2.2. Data and Blood Collection. The following data was obtained from each subject by trained personnel: weight and height (without shoes and wearing light outdoor clothing) were measured in order to calculate body mass index (BMI), waist circumference (WC), and systolic and diastolic blood pressure (SBP and DBP), the latter of which were assessed in the sitting position after a $5 \mathrm{~min}$ rest. Blood samples were collected by venopuncture from the subjects after an overnight fasting period, via both EDTA-containing tubes and tubes without anticoagulant, in order to obtain plasma, buffy-coat, and serum, and processed within 2 hours of collection. Aliquots were immediately stored at $-80^{\circ} \mathrm{C}$ until assayed.

\subsection{Laboratory Procedures}

2.3.1. Lipid Profile. Serum total cholesterol (total-c), HDL cholesterol (HDL-c), LDL cholesterol (LDL-c), and triglycerides (TGs) were analysed on a Hitachi 717 analyser (Roche Diagnostics) using standard laboratorial methods. Totalc reagents and TGs kit were obtained from bioMérieux SA (Lyon, France). HDL-c plus and LDL-c plus tests were obtained from F. Hoffmann-La Roche Ltd. (Roche Diagnostics Div., Basel, Switzerland). Serum glucose levels were measured using a glucose oxidase commercial kit (Sigma, St. Louis, Mo, USA). Plasma concentration of Ox-LDL was evaluated by using a standard commercial enzyme-linked immunoassay (Oxidized LDL ELISA, Mercodia, Uppsala, Sweden) (intra- and interassay precision of $<7.3 \%$ and $<6.2 \%$, resp.).

2.3.2. HDL Subpopulations Assay. Subpopulations were separated and quantified using a Lipoprint kit from Quantimetrix Corp. (Redondo Beach, CA, USA). The assay involves a polyacrylamide gel electrophoresis assay and a complete Lipoprint System for data acquisition and quantification of large, intermediate, and small subpopulations of HDL. 
2.3.3. PON1 Paraoxonase Activity. It was assessed spectrophotometrically and expressed in nmol of p-nitrophenol/ $\mathrm{mL} / \mathrm{min}$. In brief, paraoxonase activity was measured by adding serum to $1 \mathrm{~mL}$ Tris/ $\mathrm{HCl}$ buffer $(100 \mathrm{mmol} / \mathrm{L}, \mathrm{pH} 8.0)$ containing $2 \mathrm{mmol} / \mathrm{L} \mathrm{CaCl}_{2}$ and $5.5 \mathrm{mmol} / \mathrm{L}$ paraoxon $(\mathrm{O}, \mathrm{O}-$ diethyl-O-p-nitrophenylphosphate; Sigma Chemical Co.). The rate of generation of $\mathrm{p}$-nitrophenol was determined at $412 \mathrm{~nm}, 37^{\circ} \mathrm{C}$, via the use of a continuously recording spectrophotometer (Beckman DU-68).

2.3.4. Serum Inflammatory, Angiogenic, and Endothelial Markers. Serum adiponectin, TNF- $\alpha$, and VEGF contents were assessed using Quantikine enzyme-linked immunoassays kits from R\&D Systems (Minneapolis, USA) (intraassay precision: $<4.7 \%,<8.5 \%$, and $<6.7 \%$; interassay precision: $<10.6 \%,<6.9 \%$, and $<8.8 \%$, resp.); serum intercellular adhesion molecule 1 (iCAM1) levels were evaluated by using an ElISA kit from Abcam (Cambridge, MA, USA) (intra- and interassay precision of $<10 \%$ and $<12 \%$, resp.); high-sensitivity C-reactive protein (hsCRP) was evaluated by immunoturbidimetry, using commercially available kits (CRP (latex) high sensitivity, Roche Diagnostics); uric acid was analyzed on a Hitachi 717 analyser (Roche Diagnostics) using standard laboratory methods.

2.4. Statistical Analysis. Statistical analysis was performed by using the IBM statistical package for social sciences (SPSS) for Windows, version 20.0, (SPSS, Inc., Chicago, IL, USA). The distribution of continuous variables was analyzed using the Kolmogorov-Smirnov tests, to assess significant departures from normality. Comparisons between groups were performed using the independent samples $t$-test and the Mann-Whitney test. Adjustment of statistical differences for confounding factors (age and BMI) was performed using analysis of covariance (ANCOVA). The association between categorical variables was analyzed using Pearson's test. Statistical significance was accepted at $P$ less than 0.05 .

\section{Results}

3.1. Anthropometric Data. The demographic and anthropometric data of volunteer subjects (men and womenpre- and postmenopause) are summarized in Table 1. One hundred and twenty-four volunteers were recruited: 56 men and 68 women (48 in the premenopausal and 20 in the postmenopausal stage). The men population presented significantly higher age and BMI than the women one (Table 1); all data was analyzed after age and BMI adjustment. Men presented significantly higher values of WC, fasting glycemia, and systolic and diastolic BP, relative to the women subjects $(P=0.000$ for all $)$.

Postmenopausal women presented significantly higher age and BMI than premenopausal women (Table 1), and data was analyzed after adjusting for both parameters. Postmenopausal women showed significantly higher values of WC, fasting glycemia, and systolic and diastolic BP $(P=$ 0.000 for all).
3.2. Classical Lipid Profile. Table 2 summarizes the results obtained for all markers of lipid, inflammatory, and angiogenic profile tested in the study. With respect to classical lipid profile, several significant differences were found between men and women volunteers: (1) total-c $(P=0.001)$, TGs, and LDL-c $(P=0.000$, for both) serum content were significantly higher in men, and (2) serum HDL-c concentrations were significantly $(P=0.000)$ lower (Figure 1$)$. Despite these differences, it must be emphasized that, with the exception of total-c (slightly above acceptable limits), all of these values were within the normal range.

Concerning the effects of menopause, the following significant differences were observed: (1) serum LDL-c contents were significantly $(P=0.005)$ higher in postmenopausal women, and (2) HDL-c levels were significantly $(P=0.001)$ lower (Figures 1(a) and 1(c), resp.). No statistically significant differences were found between both groups with respect to total-c and TGs levels (Table 2).

3.3. Ox-LDL, HDL Subpopulations, and Paraoxonase Activity. Nonclassical markers of lipid profile confirm the notoriously protective status of women (and specifically, premenopausal women): Ox-LDL content is significantly higher in men and postmenopausal groups $(P=0.000$ and $P=0.009$, resp.) (Figure 1(b)). The concentration of the more protective HDL subpopulation (large HDL) was significantly higher in women (as opposed to men) and premenopausal (as opposed to postmenopausal) women $(P=0.000$ and $P=$ 0.014 , resp.) (Figure 1(d)). No differences were found between groups with regard to the content of the "less protective" HDL subpopulation (small HDL) or of intermediate HDL (Figure 1(d)). The increased CVR in men is also manifested by the higher Ox-LDL/LDL-c ratio $(P=0.000)$, while no statistical significant differences were found between the preand postmenopausal groups (Table 2). PON1 activity was lower in women $(P=0.008)$ and unchanged between preand postmenopausal subgroups.

3.4. Markers of Inflammation, Angiogenesis, and Endothelial Lesion. TNF- $\alpha$ levels were higher $(P=0.000)$ in men than in women, a tendency that was coupled with decreased $(P=$ 0.015 ) adiponectin contents (Table 2 ), with values unchanged for pre- and postmenopausal subjects. hsCRP contents were higher in women $(P=0.019)$ relative to men and in postmenopausal $(P=0.002)$ relative to premenopausal subjects. Uric acid concentration was higher in men $(P=$ 0.000 ), but no differences were found between the pre- and postmenopausal subjects. Values for the marker of endothelial lesion (iCAM-1) were found to be unchanged across groups. VEGF serum levels were significantly increased in postmenopausal women $(P=0.047)$, with unchanged levels across gender (Table 2).

\subsection{Analysis of Correlations between Markers of CVR}

3.5.1. Ox-LDL Correlations with HDL Subpopulations. A significant negative correlation was found between large HDL-c and Ox-LDL for men and women (Figure 2(b1)), 
TABLE 1: Demographic and anthropometric data of the study groups.

\begin{tabular}{|c|c|c|c|c|c|c|}
\hline Parameters & $\begin{array}{c}\text { Men } \\
\text { population } \\
(n=56)\end{array}$ & $\begin{array}{c}\text { Women } \\
\text { population } \\
(n=68)\end{array}$ & $P$ & $\begin{array}{l}\text { Premenopause } \\
\qquad(n=48)\end{array}$ & $\begin{array}{l}\text { Postmenopause } \\
\quad(n=20)\end{array}$ & $P$ \\
\hline Age, years (range) & $\begin{array}{c}53.07 \pm 1.90 \\
(22-75)\end{array}$ & $\begin{array}{c}43.16 \pm 1.81 \\
(21-69)\end{array}$ & 0.000 & $\begin{array}{c}35.40 \pm 1.39 \\
(21-54)\end{array}$ & $\begin{array}{c}61.80 \pm 1.40 \\
(49-69)\end{array}$ & 0.000 \\
\hline BMI, $\mathrm{Kg} / \mathrm{m}^{2}$ & $27.55 \pm 0.61$ & $25.28 \pm 0.50$ & 0.003 & $24.51 \pm 0.59$ & $27.14 \pm 0.81$ & 0.016 \\
\hline Waist circumference, $\mathrm{cm}$ & $99.50 \pm 1.53$ & $89.16 \pm 1.32$ & 0.000 & $87.57 \pm 1.48$ & $93.08 \pm 2.60$ & 0.000 \\
\hline SBP, mmHg & $141.77 \pm 2.57$ & $131.95 \pm 2.82$ & 0.000 & $123.04 \pm 2.59$ & $154.00 \pm 4.38$ & 0.000 \\
\hline DBP, $\mathrm{mmHg}$ & $85.36 \pm 1.40$ & $82.85 \pm 1.51$ & 0.000 & $79.74 \pm 1.70$ & $90.53 \pm 2.41$ & 0.000 \\
\hline Glycemia, mmol/L & $5.44 \pm 0.08$ & $4.94 \pm 0.06$ & 0.000 & $4.78 \pm 0.06$ & $5.33 \pm 0.14$ & 0.000 \\
\hline
\end{tabular}

Results are presented as mean \pm SEM. $P$ values adjusted for age and BMI. BMI: body mass index; SBP: systolic blood pressure; DBP: diastolic blood pressure.

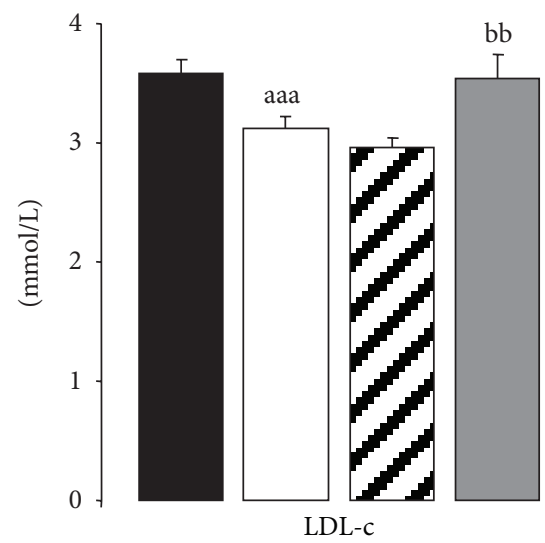

(a)

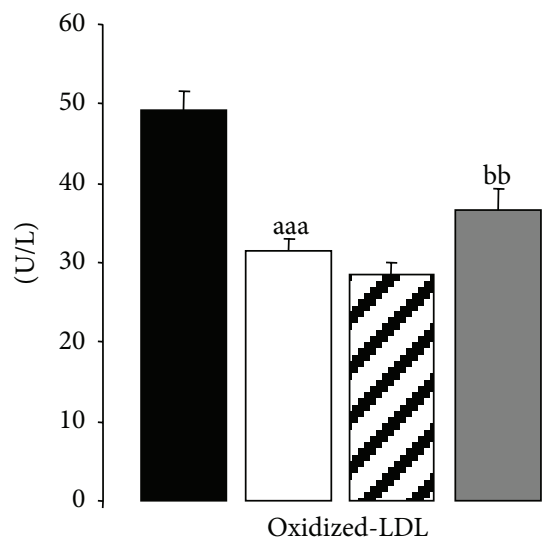

(b)

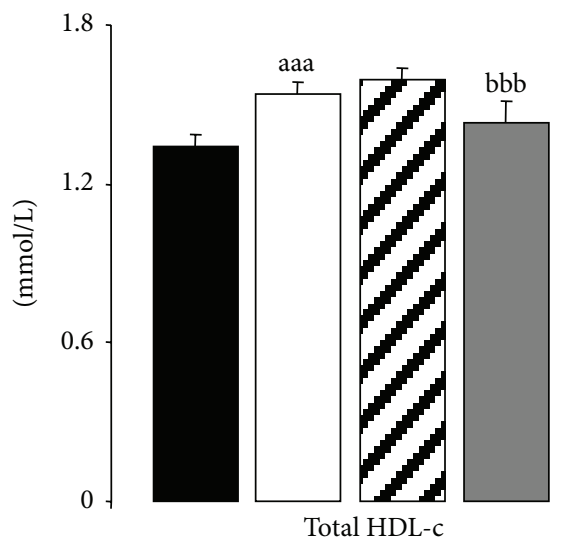

(c)
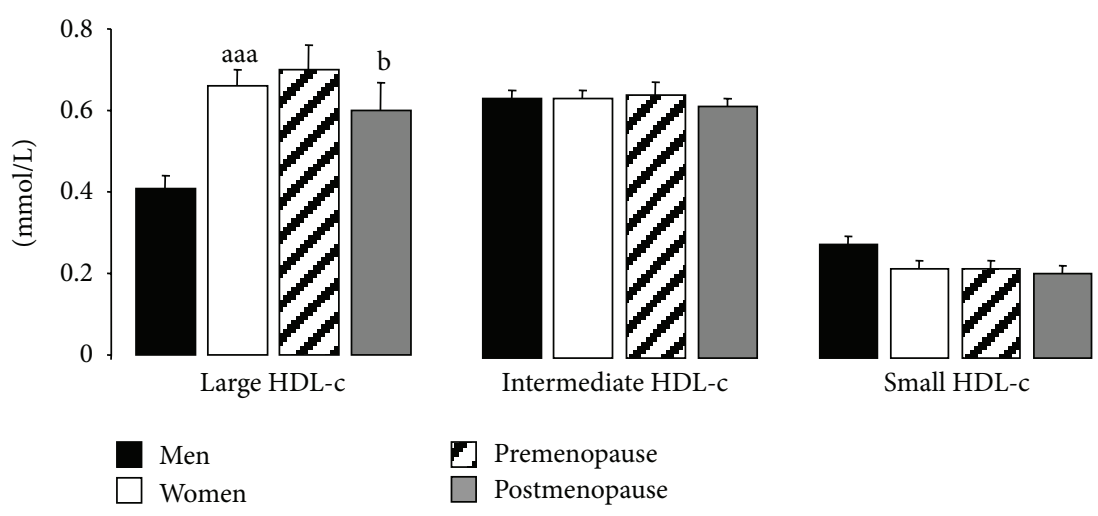

(d)

FIgURE 1: Serum LDL-c (a), Ox-LDL (b), total HDL-c (c), and large, intermediate, and small HDL subpopulations (d), in the study groups. Results are presented as mean \pm SEM. $P$ values adjusted for age and BMI. $\mathrm{a}=P<0.05$ and aaa $=P<0.001$ versus men; $\mathrm{b}=P<0.05, \mathrm{bb}=$ $P<0.01$ and $\mathrm{bbb}=P<0.001$ versus premenopause.

and postmenopausal (Figure 2(b2)) volunteers $(r=-0.288$, $P=0.045 ; r=-0.366, P=0.008 ; r=-0.570$, $P=0.011$, resp.); a significant positive correlation was also established between small HDL-c and Ox-LDL for the same groups $(r=0.306, P=0.033 ; r=0.324$, $P=0.020 ; r=0.579, P=0.009$, resp.) (Figures 2(c1) and 2(c2), resp.). No significant correlations were found between these parameters for the premenopausal group (Figure 2(b2) and 2(c2), resp.). Moreover, total
HDL-c showed no correlation with Ox-LDL in any study group (Figures 2(a1) and 2(a2)).

3.5.2. TNF- $\alpha$ Correlations with Lipid Parameters. Analysing the correlation between TNF- $\alpha$ and (resp.) total HDL-c, large HDL-c, and small HDL-c, a significant negative correlation was found between TNF- $\alpha$ and both total HDL-c and large HDL-c in women and (resp.) postmenopausal (Figures 3(a1), 


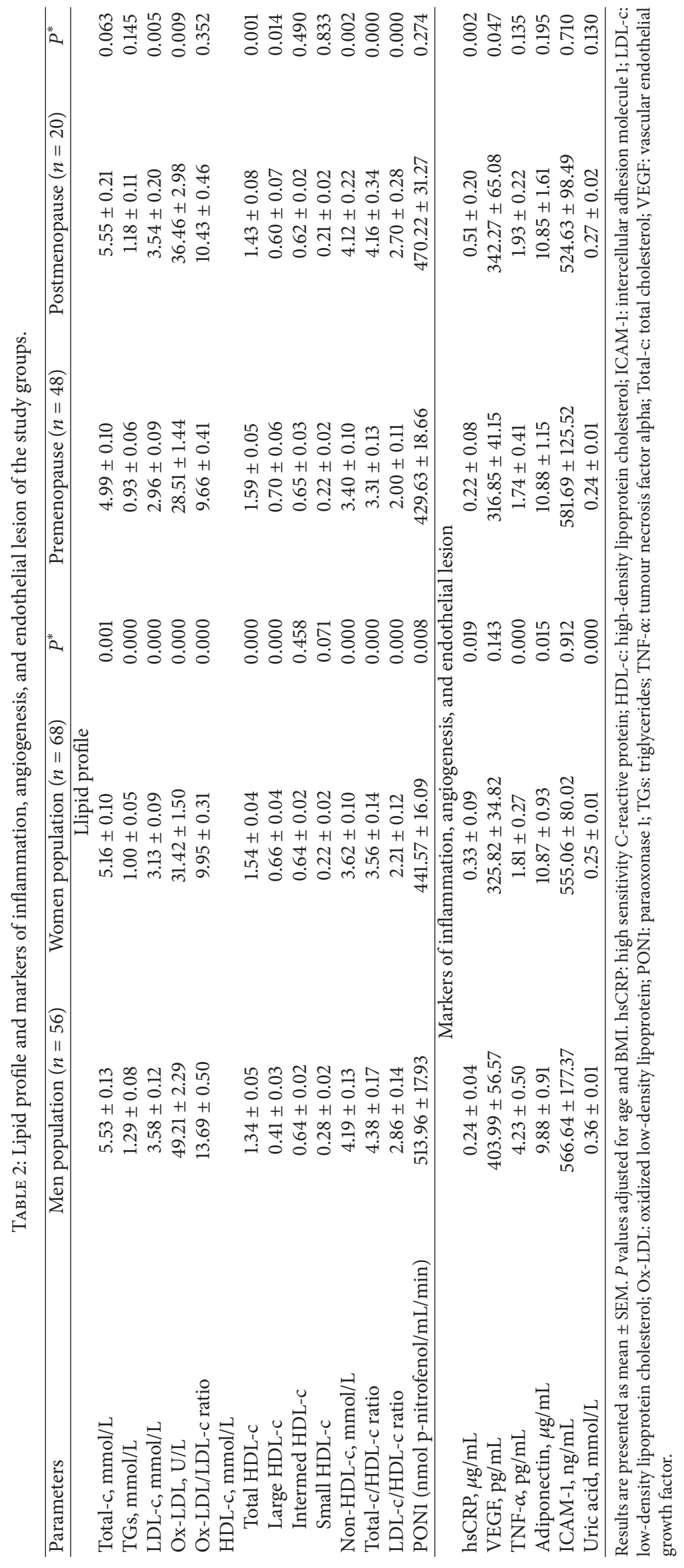




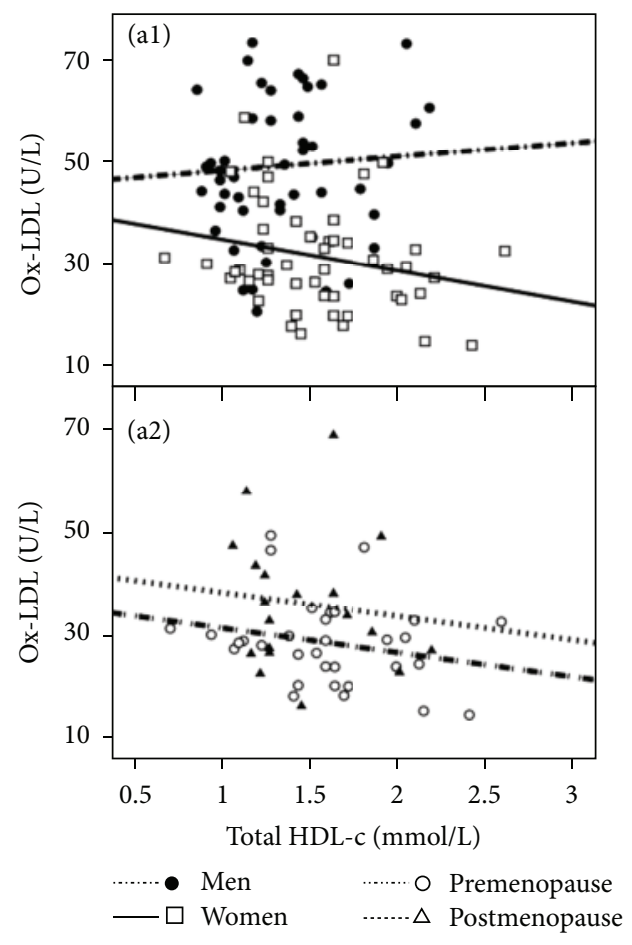

(a)

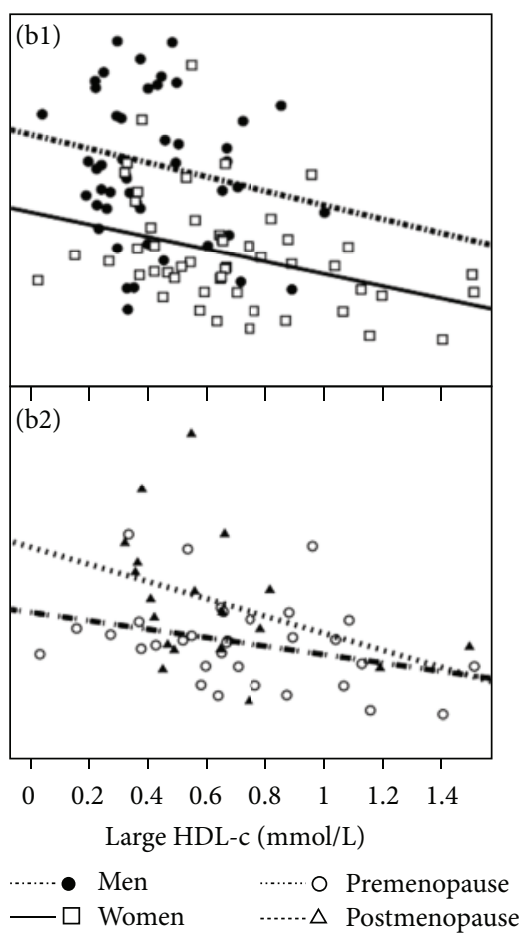

(b)

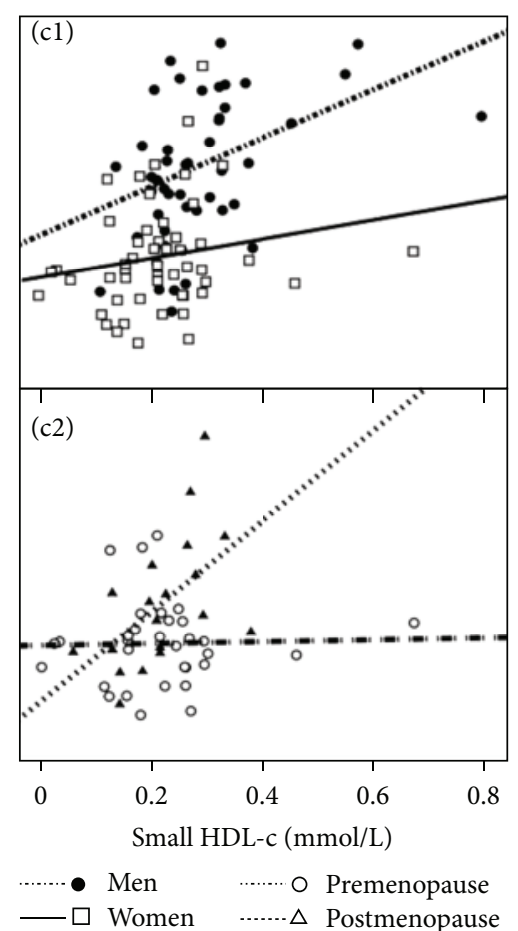

(c)

Figure 2: Correlation of Ox-LDL with total HDL-c (a), large HDL-c (b), and small HDL-c (c). Upper panel—gender influence (men versus women); lower panel-menopause influence (pre-versus postmenopause). (al) men: $r=0.058, P=0.690$; (al) women: $r=-0.221, P=0.118$; (b1) men: $r=-0.288^{*}, P=0.045$; (b1) women: $r=-0.366^{* *}, P=0.008$; (c1) men: $r=0.306^{*}, P=0.033$; (c1) women: $r=0.324^{*}, P=0.020$; (a2) premenopause: $r=-0.239, P=0.188$; (a2) postmenopause: $r=-0.119, P=0.626$; (b2) premenopause: $r=-0.255, P=0.159$; (b2) postmenopause: $r=-0.570^{*}, P=0.011$; (c2): premenopause: $r=0.114, P=0.535$; (c2) postmenopause: $r=0.579^{* *}, P=0.009$.

3(a2), 3(b1) and 3(b2), resp.) volunteers $(r=-0.345$, $P=0.013 ; r=-0.325, P=0.175 ; r=-0.031, P=0.830 ; r=$ $-0.489, P=0.033$, resp.) and a significant positive correlation between TNF- $\alpha$ and small HDL-c in postmenopausal women $(r=0.658, P=0.002)$ (Figure 3(c2)). Regarding LDL-c and $\mathrm{Ox}-\mathrm{LDL}$, the first parameter showed a significant positive correlation with TNF- $\alpha$ in the women group $(r=0.283$, $P=0.044$ ) (Figure 3(e1)) but no significant correlations in the other study groups; Ox-LDL presented a significant positive correlation with TNF- $\alpha$ in both men and postmenopausal women $(r=0.612, P=0.000$ and $r=0.570, P=0.011$, resp.) (Figures $3(\mathrm{~d} 1)$ and $3(\mathrm{~d} 2))$.

\section{Discussion}

The main finding of this study is that men and postmenopausal women volunteers present an increased CVR, which is better diagnosed when analyzed in terms of nontraditional markers. Across genders, the increased CVR in men is hidden behind an almost-normal classic lipid panel, reinforcing the importance of new biochemical markers. In fact, even though the contents of total-c, LDL-c, and TGs were significantly higher, and those of HDL-c significantly lower, in men (versus women), all values were within the normal range. Since previous studies have described the existence of lifetime variation of lipid profile measures according to age (in both genders) $[15,16]$, our results of comparisons between groups were obtained with adjustment for age in order to eliminate this putative confounding factor. In addition to the classical lipid profile parameters, men volunteers also presented higher values for Ox-LDL content and Ox-LDL/LDLc ratio, as well as lower large HDL-c concentration, all of which are lipid profile parameters not considered classically. Combined hyperlipidemia (i.e., elevated LDL-c and TGs) has been shown to increase the risk of coronary heart disease (CHD) to a significantly greater extent than either high LDLc or TGs alone, which might result from an additive effect on LDL oxidation [17]. In our study, despite both parameters being in the normal range in men subjects, they presented higher values of these parameters than women, a fact that was coupled with a concomitant increase in Ox-LDL. Relative to women, men also presented significantly increased BMI, WC, and glycemia, which seemingly also contribute to elevated Ox-LDL, as suggested in other studies [18-20]. Thus, slight (coupled) increases of blood lipids and glucose, as well as obesity, while within the normal clinical range, could create a prooxidative condition leading to LDL and HDL oxidation, as manifested in the men subjects.

While low levels of HDL-c are associated with increased CAD risk [21], a recent trial suggests that high serum HDLc content is not equally atheroprotective [22]. Rather, it has 


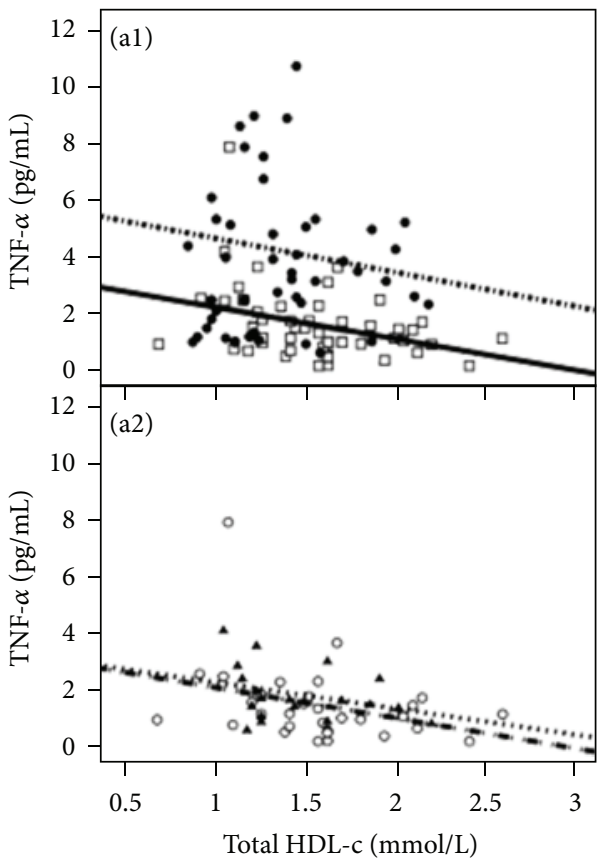

(a)

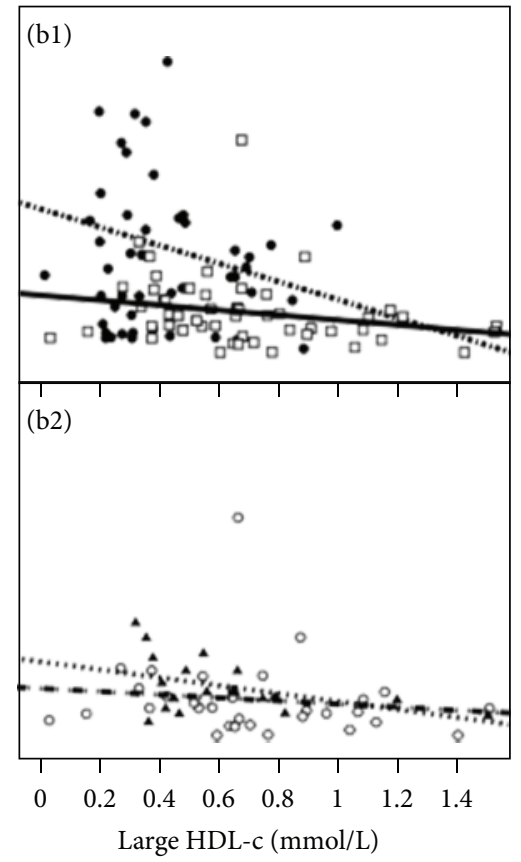

(b)

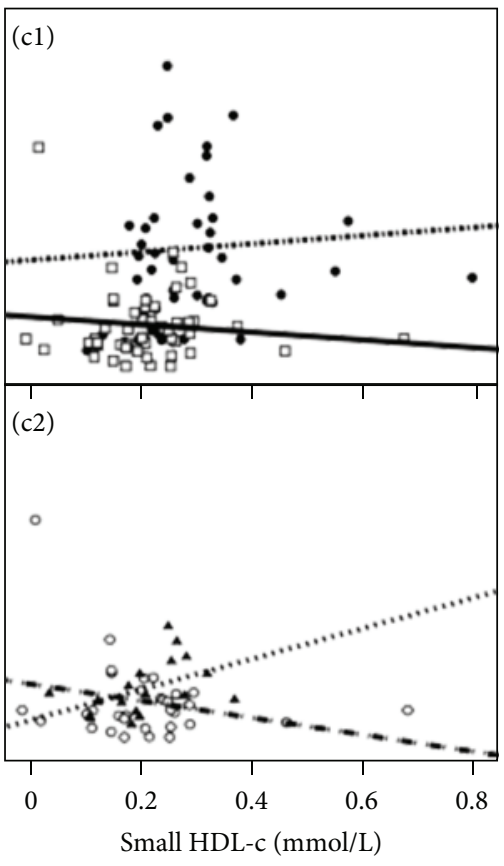

(c)

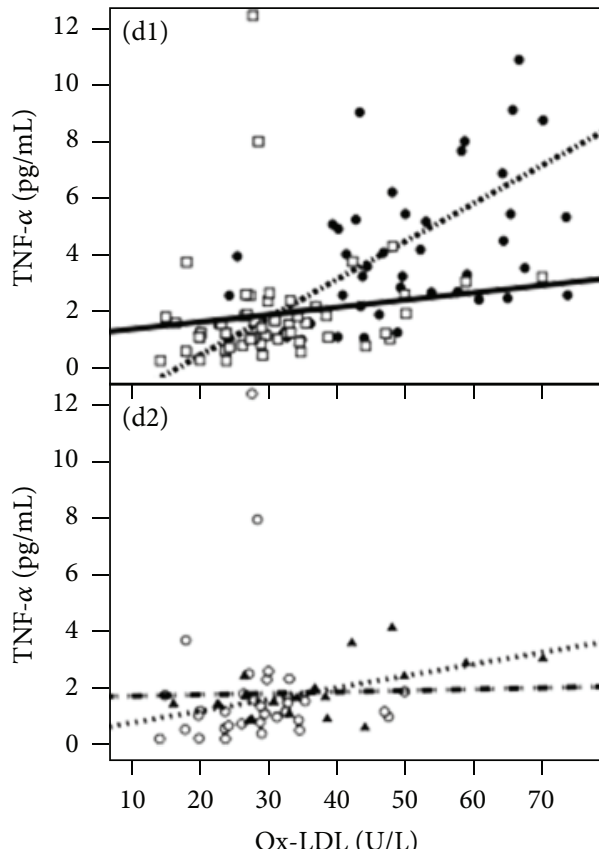

Ox-LDL (U/L)
…… O Premenopause ..... $\Delta$ Postmenopause

(d)

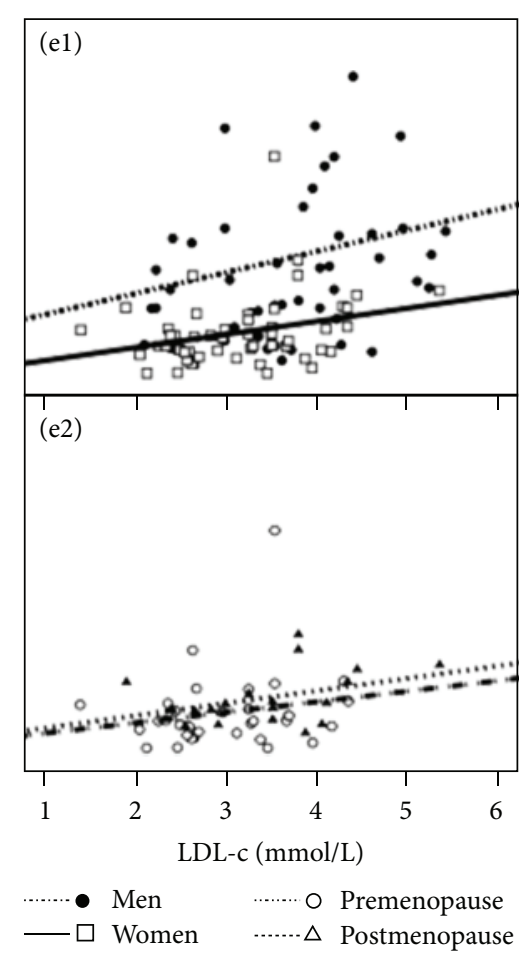

(e)

FIGURE 3: Correlation of TNF- $\alpha$ with total HDL-c (a), large HDL-c (b), small HDL-c (c), LDL-c (d), and Ox-LDL (e). Upper panel-gender influence (men versus women); lower panel-menopause influence (pre- versus postmenopause). (al) men $r=-0.119, P=0.410$; (a1) women: $r=-0.345^{*}, P=0.013$; (b1) men: $r=-0.221, P=0.122$; (b1) women: $r=-0.031, P=0.830$; (cl) men: $r=0.148, P=0.305$; (c1) women: $r=0.025, P=0.862$; (d1) men: $r=0.612^{* * *}, P=0.000$; (d1) women: $r=0.146, P=0.301$; (el) men: $r=0.193, P=0.180$; (el) women: $r=0.283^{*}, P=0.044$. (a2) premenopause: $r=-0.325, P=0.070$; (a2) postmenopause: $r=-0.325, P=0.175$; (b2) premenopause: $r=0.126, P=0.491$; (b2) postmenopause: $r=-0.489^{*}, P=0.033$; (c2) premenopause: $r=-0.222, P=0.222$; (c2) postmenopause: $r=0.658^{* *}, P=0.002$; (d2) premenopause: $r=0.017, P=0.924$; (d2) postmenopause: $r=0.570^{*}, P=0.011$; (e2) premenopause: $r=0.186$, $P=0.308$; (e2) postmenopause: $r=0.380, P=0.108$. 
been suggested that a better indicator of functionality may be HDL quality $[23,24]$, which depends on its subpopulations' type (large versus small) and constituents, including PON1 activity $[25,26]$. Our results are in agreement with this theory: indeed, the beneficial HDL profile found in women, relative to men, was reinforced by significantly enlarged content of large HDL and a decrease in small HDL. Thus, HDL-c values in men (while within the normal range) are associated with a seemingly less protective subpopulation typology. Interestingly, while no significant correlations were found between total HDL-c versus Ox-LDL content in men or women, there were significant correlations between Ox-LDL and both large HDL-c (negatively correlated) and small HDLc (positively correlated) in each group, thereby supporting the notion that the typology of HDL subpopulations is a more relevant marker than traditional total HDL-c content. The HDL-containing enzyme PON1 has been associated with antioxidant and antiatherogenic properties, and is reduced in some populations of increased CVR $[25,26]$. In our study, women presented decreased PON1 activity, despite their increased HDL-c levels, which is in opposition with previous data that showed a slightly higher value [27]. However, PON1 activity is modulated by different aspects, including diet, smoking, and exercise habits [28-30]. In fact, as an important CVR factor, smoking habits could also modulate other mediators or factors involved in the development of CVD, including BMI, hsCRP, oxidized lipoproteins, and HDL-c. In agreement, future works will analyse this influence.

Apart from the nontraditional markers of lipid profile (in particular Ox-LDL and HDL subpopulations), men subjects also presented a proinflammatory pattern, which is a major contributor for the development of atherogenesis. Indeed, men population showed higher serum concentration of TNF$\alpha$ and uric acid, accompanied by decreased adiponectin levels. The reduced plasma cytokine concentrations, including IL- 6 and TNF- $\alpha$, in women have been attributed to the inhibitory effect of estrogens on the expression of inflammatory marker genes [31-33]. The body fat distribution, particularly abdominal visceral and subcutaneous adiposity, has been associated with gender-related variation in inflammatory markers, including hsCRP, IL-6, TNF- $\alpha$, and adiponectin. In fact, fat distribution is known to differ across genders, with men having more visceral and less subcutaneous fat [34]. In our study, even after having adjusted for BMI, we cannot exclude a contribution of abdominal visceral adiposity in men, as manifested by increased WC, for the more prejudicial inflammatory status found relative to women population, which included not only higher TNF$\alpha$ content but also lower levels of adiponectin. Adiponectin is synthesized mainly in subcutaneous fat cells-of which women have more than men-and it is secreted mostly by adipocytes, while inflammatory cytokines are secreted mainly from nonfat cells in adipose tissue [35, 36], which is in agreement with our data. In fact, other authors have suggested that the visceral adipose tissue is responsible for release factors (including TNF- $\alpha$ ) that inhibit adiponectin synthesis from the subcutaneous adipocytes [37-39], which is in agreement with our data and hypothesis of men having more visceral adipose tissue (as the increased WC suggests), as well as increased TNF- $\alpha$ content and, consequently, lower adiponectin. Interestingly, while in the women group there was a significant positive correlation between TNF- $\alpha$ content and total HDL-c, as well as a significant inverse correlation with LDL-c, in men population TNF- $\alpha$ concentration was better correlated with Ox-LDL levels, which were significantly higher in men than in women and which in turn supports the notion that Ox-LDL is a good marker of risk in men and that oxidative damage of lipoproteins is associated with increased inflammation. Indeed, Ox-LDL has already been suggested as a better marker of risk for other conditions [40-42].

Chronic inflammation and endothelial dysfunction, as observed in patients with increased serum uric acid (SUA), are likely to intervene in the mechanisms through which SUA affects renal structure and operation [43], thereby leading to the development of hypertension in (especially young) individuals [44]. Conversely, it has been questioned if uric acid increases as a defense mechanism to counteract the increased oxidative stress associated with insulin resistance, inflammation, and other risk factors that usually cluster with increased SUA concentrations [45]. Many studies show a stronger correlation between SUA and cardiovascular risk in women, thereby justifying the need for a gender-based evaluation of this correlation. On the other hand, metabolic syndrome was positively associated with increasing SUA, regardless of gender. Abdominal obesity and hypertriglyceridemia were the main factors associated with hyperuricemia even in normotensive individuals, and they may add a higher risk for hypertensive individuals [46]. Our data is in agreement with each of these observations, as it shows a simultaneous increase in uric acid, TGs, waist circumference and blood pressure in men relative to women. Whether the increased uric acid in men is a compensatory protective mechanism against inflammation and oxidation, or a contributor to the increased cardiometabolic profile, remains to be clarified.

While assessing the influence of menopause on CVR, we found that nontraditional markers once again play a key role in distinguishing between pre- and postmenopausal women. Indeed, the relatively larger concentration of LDL$c$ and the lower concentration of HDL-c associated with the postmenopausal state seem to be of little clinical significance, since in each case values are within the normal range, and no differences were found in total-c and TGs levels. However, Ox-LDL content was higher and large HDL-c lower in postmenopausal women, a trend that was coupled with increased hsCRP, thereby demonstrating a dyslipidaemic and proinflammatory profile. Furthermore, postmenopausal women presented significantly increased BMI, waist circumference, blood pressure, and glycemia, without a typical profile of dyslipidaemia, and these trends seem to be better expressed by nonclassical parameters, such as oxidized LDL (as opposed to the typically used LDL-c content) and large HDL-c (as opposed to total HDL-c content). Interestingly, Ox-LDL concentration has no correlation with total-c content in postmenopausal women but was strongly significantly correlated with large HDL-c (inversely) and small HDL-c (directly), which underlines the relevance of those 
parameters in postmenopausal risk determination. Thus, menopause seems to cause a reduction of absolute HDLc levels but more importantly changes in the composition of HDL particles. Such a conclusion is in agreement with Eapen et al. [47] who reported an alteration in the distribution of HDL subspecies in menopause, with a reduction seen in the proportion of large, buoyant HDL2 particles, which are believed to be more active in reverse cholesterol transport [48]; in contrast, the number of smaller, more dense HDL3 subfragments increases $[49,50]$. In addition, ex vivo biochemical analysis of lipoproteins from pre- and postmenopausal women suggests that the postmenopausal HDL particle exhibits impaired ability to limit LDL oxidation [51]. Despite the impaired composition of HDL particles in postmenopausal women, we did not find differences in PON1 activity. It has been pointed out that the latter HDLassociated enzyme has protective effects against inflammation in the arterial wall and that it is capable of destroying the biologically active lipids in mildly oxidized LDL; several studies have previously reported that $\mathrm{CHD}$ is associated with low PON activity $[52,53]$. In our data, however, PON1 activity is unchanged in postmenopausal women, which is in agreement with previous data from Zago et al. [51], who also found no differences in pre- versus postmenopausal women, as well as with the study of Horter et al. [54], who failed to find any significant association between $\mathrm{CHD}$ and paraoxonase or arylesterase activity in postmenopausal women.

Adipocytokine levels have previously been associated with estrogens, suggesting that menopause impacts on inflammatory mediators [55]. In our study, although the TNF- $\alpha$ increase in postmenopausal women (relative to premenopausal women) did not achieve statistical difference, the values presented strongly significant correlations with the previous markers: namely, a positive correlation with large HDL-c and Ox-LDL and an inverse correlation with small HDL-c. Surprisingly, no correlation was found between TNF- $\alpha$ levels and total HDL-c and LDL-c concentrations, which are the clinically checked parameters. Moreover, no correlation was found between TNF- $\alpha$ and either HDLc contents (total or subpopulations) or LDL-c levels (oxidized or nonoxidized measures) in premenopausal women, thereby demonstrating that the nontraditional parameters gain particular relevance after menopause and are deserving of closer attention in postmenopausal women. With regard to other mediators/markers of the inflammatory process, we found a significant increased concentration of hsCRP in the postmenopausal women. Previous studies on menopause and inflammation have showed that obesity is associated with an inflammatory state that is often characterized by elevated plasma hsCRP levels [56]. In our study, hsCRP was significantly increased in postmenopausal women, who also presented excess weight/obesity. hsCRP is an important marker for this condition, because after menopause the consequent decrease in estrogen levels contributes to widespread weight gain in women; furthermore, increased contents of hsCRP, a marker of systemic inflammation, are associated with increased incidence of CVD, independently of other conventional risk factors [57]. By contrast, adiponectin levels of the pre- and postmenopausal groups were the same, and adiponectin was uncorrelated with age, in agreement with a study of Goodarzi et al. [58], but in disagreement with Loucif et al. [59]. So it seems that menopause impacts less on adiponectin as a mediator, suggesting that women have higher adiponectin contents than men, irrespective of their menopausal state. A similar association was found for TNF$\alpha$, with women having significantly lower concentrations, whether before or after menopause.

VEGF serum levels are lower in premenopausal than postmenopausal women, which is in disagreement with the data from another study [59]. It should be noted that none of the volunteers entering in our study were under hormone replacement therapy (HRT), which could influence the levels of VEGF, although published studies are contradictory on this effect $[60,61]$.

The data obtained suggest that men subjects present an increase CV risk when compared to women which is mainly viewed by nontraditional markers and might result from slight, but concomitant, variations of several factors (despite values within the normal clinical range), including increment of TC, LDL-c, TG, and glycemia and reduced HDLc, together with increased BMI. The accumulation of factors seems to promote an oxidative and inflammatory profile, with increased contents of Ox-LDL and reduced of largeHDL-c, as well as of the proinflammatory cytokine TNF$\alpha$, and reduced of anti-inflammatory adiponectin. This cluster of procardiometabolic alterations (even of slight intensity), together with increased uric acid and blood pressure, might represent an enhanced CV risk when compared with women subjects, which seem to be protected, namely, before menopause. Similarly, after menopause, women present an increased BMI, waist circumference, and glycemia, which might underlie the modifications of cardiometabolic profile. Indeed, postmenopausal women, despite only slightly higher values of LDL-c and lower of HDL-c (an unchanged of TC and TGs), presented an oxidative, angiogenic, and proinflammatory profile, with increased contents of $\mathrm{Ox}$ LDL and VEGF and reduced of large HDL-c, together with increased hsCRP, suggesting once again that a cluster of slight variations of traditional markers (despite in the normal clinical range) promotes a poor cardiometabolic profile.

To conclude, in a population without previous diagnosis and medication for CVD, as well as without familiar history of CVD, and with serum levels of traditional routine lipid measures and glycemia within the normal range, there are nontraditional measures related with lipid, inflammatory, and angiogenic profiles that cannot be minimized and would deserve attention, as they show an increased CVR in men and in postmenopausal women. Although the issue is not consensual when refers to factors that might affect the CVR in a healthy population, this data suggests more attention to nonclassical parameters as putative biomarkers of the CV status in a population without any noticeable classical signal, marker, symptom, or previous history appointment of CVD. However, the study has some limitations that deserve further research in future work: (a) the possibility of bias related with the study design and criteria for inclusion of subjects; (b) the enlargement of sample size will strength 
the results; (c) a better age and BMI matching of populations would improve the data; (d) cardiovascular outcomes (e.g., events) will allow better association between markers and CVR.

\section{Conclusions}

Men and postmenopausal women present a lipid profile indicative of an increased CVR, relative to women and premenopausal women, respectively. This increased risk may be quantified by nontraditional lipid profile markers, including (the relative content of) $\mathrm{Ox}$-LDL and HDL subpopulations, as well as markers of inflammation and angiogenesis (including TNF- $\alpha$ and adiponectin for comparisons across genders and VEGF for comparisons between pre- and postmenopausal women). In addition, postmenopausal women manifest a proatherogenic risk that should receive special attention and might warrant early pharmacotherapeutic intervention to prevent premature development of CVD. Finally, this study suggest that some of the classical markers of cardiovascular (namely, lipidic) profile, often insensitive to risk, may present normal or only slightly elevated values and still within the normal clinical range, recommending that routine biochemical data would improve with the inclusion of other reliable and specific markers with additional information of cardiometabolic risk.

\section{Conflict of Interests}

The authors report no conflict of interests.

\section{Acknowledgments}

This study was supported by the Portuguese Foundation for Science and Technology, through a Ph.D. Grant (SFRH/BD/ 65483/2009) and Strategic Project (PEst-C/SAU/UI3282/ 2011), as well as by the COMPETE.

\section{References}

[1] C. Leuzzi, R. Marzullo, and M. G. Modena, "Is menopause a risk factor for ischemic heart disease in women?" Giornale Italiano di Cardiologia, vol. 13, no. 6, pp. 401-406, 2012.

[2] M. Kaushik, S. P. Sontineni, and C. Hunter, "Cardiovascular disease and androgens: a review," International Journal of Cardiology, vol. 142, no. 1, pp. 8-14, 2010.

[3] E. Casiglia, V. Tikhonoff, S. Caffi et al., "Menopause does not affect blood pressure and risk profile, and menopausal women do not become similar to men," Journal of Hypertension, vol. 26, no. 10, pp. 1983-1992, 2008.

[4] H. Smulyan, R. G. Asmar, A. Rudnicki, G. M. London, and M. E. Safar, "Comparative effects of aging in men and women on the properties of the arterial tree," Journal of the American College of Cardiology, vol. 37, pp. 1374-1380, 2001.

[5] H. Mangge, G. Almer, M. Truschnig-Wilders, A. Schmidt, R. Gasser, and D. Fuchs, "Inflammation, adiponectin, obesity and cardiovascular risk," Current Medicinal Chemistry, vol. 17, no. 36, pp. 4511-4520, 2010.
[6] S.-S. Huang, P.-H. Huang, Y.-H. Chen, K.-H. Chiang, J.-W. Chen, and S.-J. Lin, "Association of adiponectin with future cardiovascular events in patients after acute myocardial infarction," Journal of Atherosclerosis and Thrombosis, vol. 17, no. 3, pp. 295303, 2010.

[7] F. Girardi, E. Franceschi, and A. A. Brandes, "Cardiovascular safety of VEGF-targeting therapies: current evidence and handling strategies," Oncologist, vol. 15, no. 7, pp. 683-694, 2010.

[8] A. N. N. Mertens and P. Holvoet, "Oxidized LDL and HDL: antagonists in atherothrombosis," FASEB Journal, vol. 15, no. 12, pp. 2073-2084, 2001.

[9] M. I. Mackness, P. N. Durrington, and B. Mackness, “The role of paraoxonase 1 activity in cardiovascular disease: potential for therapeutic intervention," American Journal of Cardiovascular Drugs, vol. 4, no. 4, pp. 211-217, 2004.

[10] K. Mahdy Ali, A. Wonnerth, K. Huber, and J. Wojta, "Cardiovascular disease risk reduction by raising HDL cholesterolcurrent therapies and future opportunities," British Journal of Pharmacology, vol. 167, pp. 1177-1194, 2012.

[11] S. Redondo, J. Martínez-González, C. Urraca, and T. Tejerina, "Emerging therapeutic strategies to enhance HDL function," Lipids in Health and Disease, vol. 10, article 175, 2011.

[12] B. F. Asztalos, P. S. Roheim, R. L. Milani et al., "Distribution of apoA-I-containing HDL subpopulations in patients with coronary heart disease," Arteriosclerosis, Thrombosis, and Vascular Biology, vol. 20, no. 12, pp. 2670-2676, 2000.

[13] A. Pirillo, G. D. Norata, and A. L. Catapano, "High-density lipoprotein subfractions-what the clinicians need to know," Cardiology, vol. 124, pp. 116-125, 2013.

[14] World Health Organization Scientific Group, "Research on the menopause in the 1990s," WHO Technical Services Department Series 866, WHO, Geneva, Switzerland.

[15] G. M. Singh, G. Danaei, P. M. Pelizzari et al., "The age associations of blood pressure, cholesterol, and glucose: analysis of health examination surveys from international populations," Circulation, vol. 125, pp. 2204-2211, 2012.

[16] F. B. Dalpino, L. Menna-Barreto, and E. C. de Faria, "Influences of sex and age on biological rhythms of serum lipids and lipoproteins," Clinica Chimica Acta, vol. 406, no. 1-2, pp. 57-61, 2009.

[17] V. Manninen, L. Tenkanen, P. Koskinen et al., "Joint effects of serum triglyceride and LDL cholesterol and HDL cholesterol concentrations on coronary heart disease risk in the Helsinki Heart Study. Implications for treatment," Circulation, vol. 85, no. 1, pp. 37-45, 1992.

[18] S. Zelzer, N. Fuchs, G. Almer et al., "High density lipoprotein cholesterol level is a robust predictor of lipid peroxidation irrespective of gender, age, obesity, and inflammatory or metabolic biomarkers," Clinica Chimica Acta, vol. 412, no. 15-16, pp. 13451349, 2011.

[19] K. Kotani, N. Sakane, M. Ueda et al., "Oxidized high-density lipoprotein is associated with increased plasma glucose in nondiabetic dyslipidemic subjects," Clinica Chimica Acta, vol. 414, pp. 125-129, 2012.

[20] M. Maytin, J. Leopold, and J. Loscalzo, "Oxidant stress in the vasculature," Current Atherosclerosis Reports, vol. 1, no. 2, pp. 156-164, 1999.

[21] R. Movva and D. J. Rader, "Laboratory assessment of HDL heterogeneity and function," Clinical Chemistry, vol. 54, no. 5, pp. 788-800, 2008. 
[22] D. J. Rader, "Illuminating HDL-is it still a viable therapeutic target?" The New England Journal of Medicine, vol. 357, no. 21, pp. 2180-2183, 2007.

[23] E. Eren, N. Yilmaz, and O. Aydin, "High density lipoprotein and it's dysfunction," The Open Biochemistry Journal, vol. 6, pp. 7893, 2012.

[24] H. Soran, S. Hama, R. Yadav et al., "HDL functionality," Current Opinion in Lipidology, vol. 23, pp. 353-366, 2012.

[25] M. Mackness, P. Durrington, and B. Mackness, "Paraoxonase 1 activity, concentration and genotype in cardiovascular disease," Current Opinion in Lipidology, vol. 15, no. 4, pp. 399-404, 2004.

[26] M. I. Mackness, B. Mackness, and P. N. Durrington, "Paraoxonase and coronary heart disease," Atherosclerosis Supplements, vol. 3, no. 4, pp. 49-55, 2002.

[27] P. Kleemola, R. Freese, M. Jauhiainen, R. Pahlman, G. Alfthan, and M. Mutanen, "Dietary determinants of serum paraoxonase activity in healthy humans," Atherosclerosis, vol. 160, no. 2, pp. 425-432, 2002.

[28] E. Thomàs-Moyà, M. Gianotti, A. M. Proenza, and I. Lladó, "Paraoxonase 1 response to a high-fat diet: gender differences in the factors involved," Molecular Medicine, vol. 13, no. 3-4, pp. 203-209, 2007.

[29] E. Thomàs-Moyà, M. Gianotti, I. Lladó, and A. M. Proenza, "Effects of caloric restriction and gender on rat serum paraoxonase 1 activity," Journal of Nutritional Biochemistry, vol. 17, no. 3, pp. 197-203, 2006.

[30] E. Thomàs-Moyà, Y. Gómez-Pérez, M. Fiol, M. Gianotti, I. Lladó, and A. M. Proenza, "Gender related differences in paraoxonase 1 response to high-fat diet-induced oxidative stress," Obesity, vol. 16, no. 10, pp. 2232-2238, 2008.

[31] W. B. Ershler and E. T. Keller, "Age-associated increased interleukin-6 gene expression, late-life diseases, and frailty," Annual Review of Medicine, vol. 51, pp. 245-270, 2000.

[32] J. An, R. C. J. Ribeiro, P. Webb et al., "Estradiol repression of tumor necrosis factor- $\alpha$ transcription requires estrogen receptor activation function- 2 and is enhanced by coactivators," Proceedings of the National Academy of Sciences of the United States of America, vol. 96, no. 26, pp. 15161-15166, 1999.

[33] S. D. Imahara, S. Jelacic, C. E. Junker, and G. E. O’Keefe, "The influence of gender on human innate immunity," Surgery, vol. 138, no. 2, pp. 275-282, 2005.

[34] B. Ludescher, A. Najib, S. Baar et al., "Gender specific correlations of adrenal gland size and body fat distribution: a whole body MRI study," Hormone and Metabolic Research, vol. 39, no. 7, pp. 515-518, 2007.

[35] J. N. Fain, "Release of interleukins and other inflammatory cytokines by human adipose tissue is enhanced in obesity and primarily due to the nonfat cells," Vitamins and Hormones, vol. 74, pp. 443-477, 2006.

[36] T. Ahonen, M. Vanhala, H. Kautiainen, E. Kumpusalo, and J. Saltevo, "Sex differences in the association of adiponectin and low-grade inflammation with changes in the body mass index from youth to middle age," Gender Medicine, vol. 9, no. 1, pp. $1-8,2011$.

[37] C. M. Halleux, M. Takahashi, M. L. Delporte et al., "Secretion of adiponectin and regulation of apM1 gene expression in human visceral adipose tissue," Biochemical and Biophysical Research Communications, vol. 288, no. 5, pp. 1102-1107, 2001.

[38] N. Maeda, M. Takahashi, T. Funahashi et al., "PPAR $\gamma$ ligands increase expression and plasma concentrations of adiponectin, an adipose-derived protein," Diabetes, vol. 50, no. 9, pp. 20942099, 2001
[39] Y. Matsuzawa, "Establishment of a concept of visceral fat syndrome and discovery of adiponectin," Proceedings of the Japan Academy. Series B, vol. 86, pp. 131-141, 2010.

[40] H. Huang, W. Mai, D. Liu, Y. Hao, J. Tao, and Y. Dong, "The oxidation ratio of LDL: a predictor for coronary artery disease," Disease Markers, vol. 24, no. 6, pp. 341-349, 2008.

[41] Y. Huang, Y. Hu, W. Mai et al., "Plasma oxidized low-density lipoprotein is an independent risk factor in young patients with coronary artery disease," Disease Markers, vol. 31, no. 5, pp. 295301, 2011.

[42] H. Huang, R. Ma, D. Liu et al., "Oxidized low-density lipoprotein cholesterol and the ratio in the diagnosis and evaluation of therapeutic effect in patients with coronary artery disease," Disease Markers, vol. 33, pp. 295-302, 2012.

[43] C. Zoccali, R. Maio, F. Mallamaci, G. Sesti, and F. Perticone, "Uric acid and endothelial dysfunction in essential hypertension," Journal of the American Society of Nephrology, vol. 17, no. 5, pp. 1466-1471, 2006.

[44] D. I. Feig and R. J. Johnson, "Hyperuricemia in childhood primary hypertension," Hypertension, vol. 42, no. 3, pp. 247-252, 2003.

[45] F. J. Nieto, C. Iribarren, M. D. Gross, G. W. Comstock, and R. G. Cutler, "Uric acid and serum antioxidant capacity: a reaction to atherosclerosis?" Atherosclerosis, vol. 148, no. 1, pp. 131-139, 2000.

[46] S. L. Rodrigues, M. P. Baldo, D. P. Capingana et al., "Gender distribution of serum uric acid and cardiovascular risk factors: population based study," Arquivos Brasileiros de Cardiologia, vol. 98, no. 1, pp. 13-21, 2012.

[47] D. J. Eapen, G. L. Kalra, L. Rifai, C. A. Eapen, N. Merchant, and B. V. Khan, "Raising HDL cholesterol in women," International Journal of Women's Health, vol. 1, no. 1, pp. 181-191, 2009.

[48] Y. Huang, A. Von Eckardstein, S. Wu, and G. Assmann, "Cholesterol efflux, cholesterol esterification, and cholesteryl ester transfer by LpA-I and LpA-I/A-II in native plasma," Arteriosclerosis, Thrombosis, and Vascular Biology, vol. 15, no. 9, pp. 1412-1418, 1995.

[49] J. C. Stevenson, D. Crook, and I. F. Godsland, "Influence of age and menopause on serum lipids and lipoproteins in healthy women," Atherosclerosis, vol. 98, no. 1, pp. 83-90, 1993.

[50] Z. Li, J. R. McNamara, J.-C. Fruchart et al., "Effects of gender and menopausal status on plasma lipoprotein subspecies and particle sizes," Journal of Lipid Research, vol. 37, no. 9, pp. 18861896, 1996.

[51] V. Zago, S. Sanguinetti, F. Brites et al., "Impaired high density lipoprotein antioxidant activity in healthy postmenopausal women," Atherosclerosis, vol. 177, no. 1, pp. 203-210, 2004.

[52] P. M. Laplaud, T. Dantoine, and M. J. Chapman, "Paraoxonase as a risk marker for cardiovascular disease: facts and hypotheses," Clinical Chemistry and Laboratory Medicine, vol. 36, no. 7, pp. 431-441, 1998.

[53] P. N. Durrington, B. Mackness, and M. I. Mackness, "Paraoxonase and atherosclerosis," Arteriosclerosis, Thrombosis, and Vascular Biology, vol. 21, no. 4, pp. 473-480, 2001.

[54] M. J. Horter, S. Sondermann, H. Reinecke et al., "Associations of HDL phospholipids and paraoxonase activity with coronary heart disease in postmenopausal women," Acta Physiologica Scandinavica, vol. 176, no. 2, pp. 123-130, 2002.

[55] S. C. Hong, S. W. Yoo, G. J. Cho et al., "Correlation between estrogens and serum adipocytokines in premenopausal and postmenopausal women," Menopause, vol. 14 , no. 5, pp. 835840, 2007. 
[56] B. J. Arsenault, C. P. Earnest, J.-P. Després, S. N. Blair, and T. S. Church, "Obesity, coffee consumption and CRP levels in postmenopausal overweight/obese women: importance of hormone replacement therapy use," European Journal of Clinical Nutrition, vol. 63, no. 12, pp. 1419-1424, 2009.

[57] J. Danesh, J. G. Wheeler, G. M. Hirschfield et al., "C-reactive protein and other circulating markers of inflammation in the prediction of coronary heart disease," The New England Journal of Medicine, vol. 350, no. 14, pp. 1387-1397, 2004.

[58] M. T. Goodarzi, H. Babaahmadi-Rezaei, M. KadkhodaeiEliaderani, and S. Haddadinezhad, "Relationship of serum adiponectin with blood lipids, HbAlc, and hs-CRP in type II diabetic postmenopausal women," Journal of Clinical Laboratory Analysis, vol. 21, no. 3, pp. 197-200, 2007.

[59] Y. Loucif, J. Méthot, K. Tremblay, D. Brisson, and D. Gaudet, "Contribution of adiponectin to the cardiometabolic risk of postmenopausal women with loss-of-function lipoprotein lipase gene mutations," Menopause, vol. 18, no. 5, pp. 558-562, 2011.

[60] R. Agrawal, G. Prelevic, G. S. Conway, N. N. Payne, J. Ginsburg, and H. S. Jacobs, "Serum vascular endothelial growth factor concentrations in postmenopausal women: the effect of hormone replacement therapy," Fertility and Sterility, vol. 73, no. 1, pp. 56-60, 2000.

[61] H. Sumino, T. Nakamura, S. Ichikawa et al., "Serum level of vascular endothelial growth factor is decreased by hormone replacement therapy in postmenopausal women without hypercholesterolemia," Atherosclerosis, vol. 148, no. 1, pp. 189-195, 2000 . 


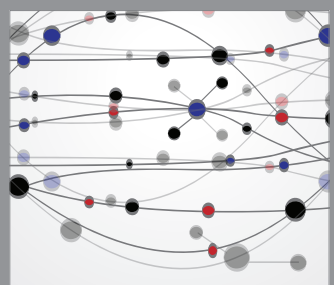

The Scientific World Journal
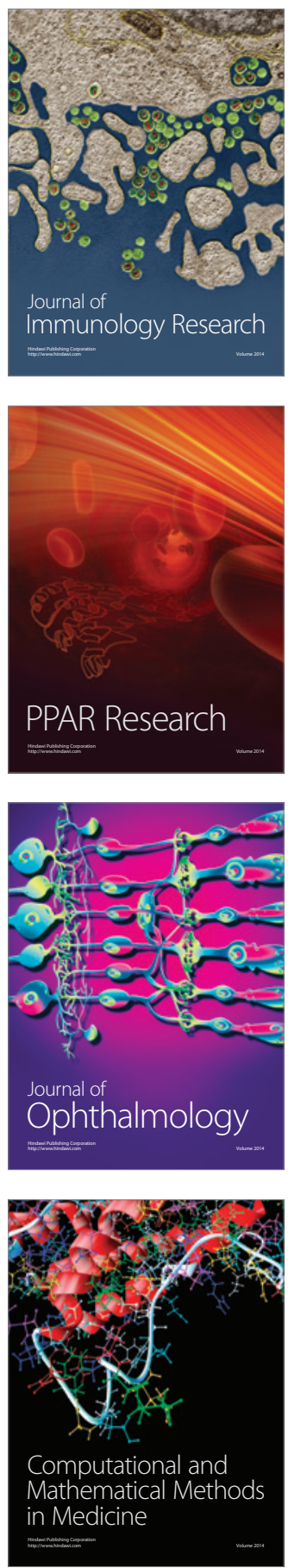

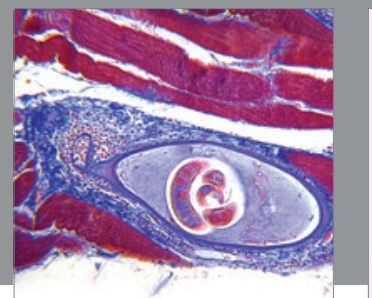

Gastroenterology

Research and Practice
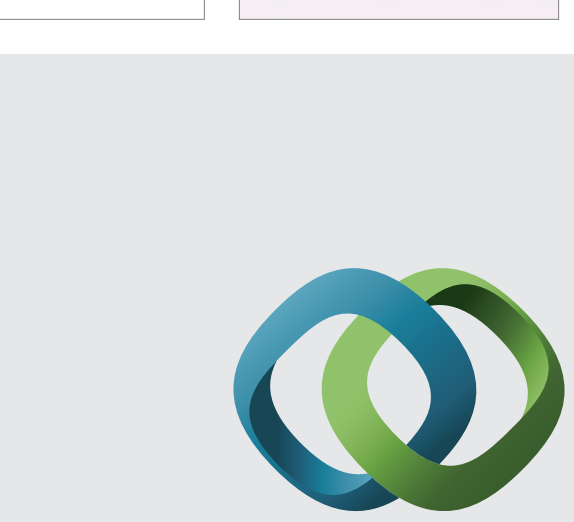

\section{Hindawi}

Submit your manuscripts at

http://www.hindawi.com
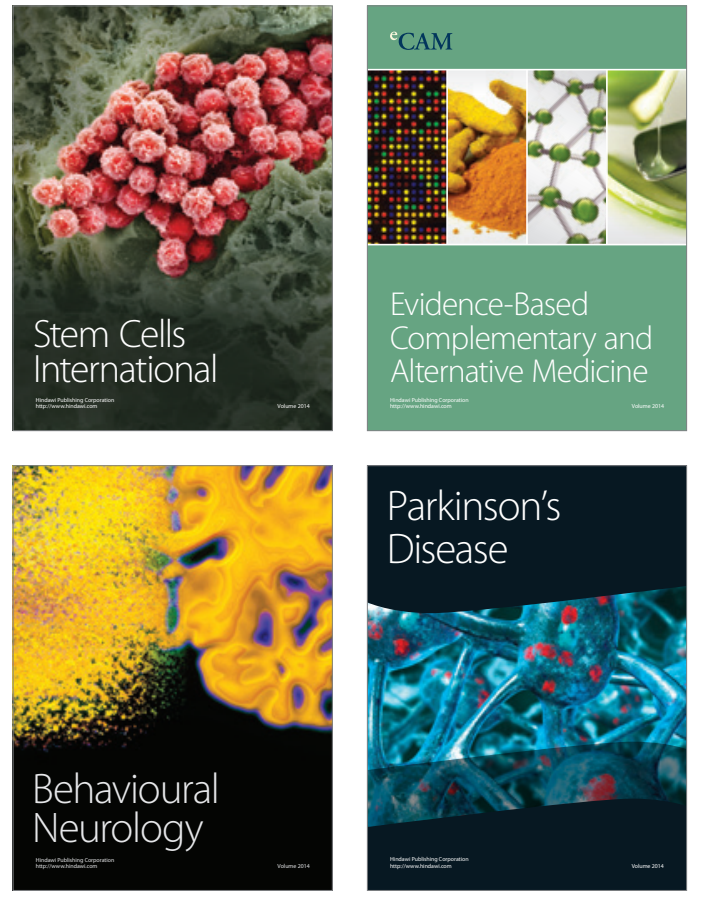
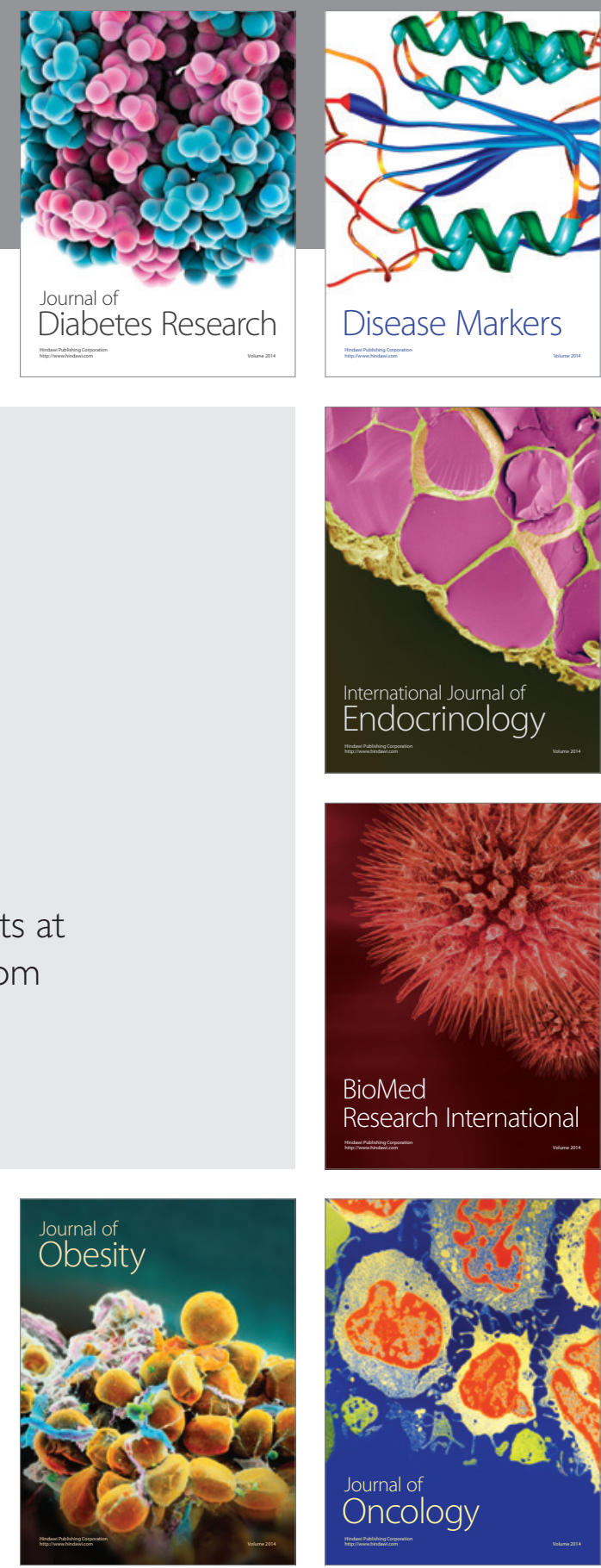

Disease Markers
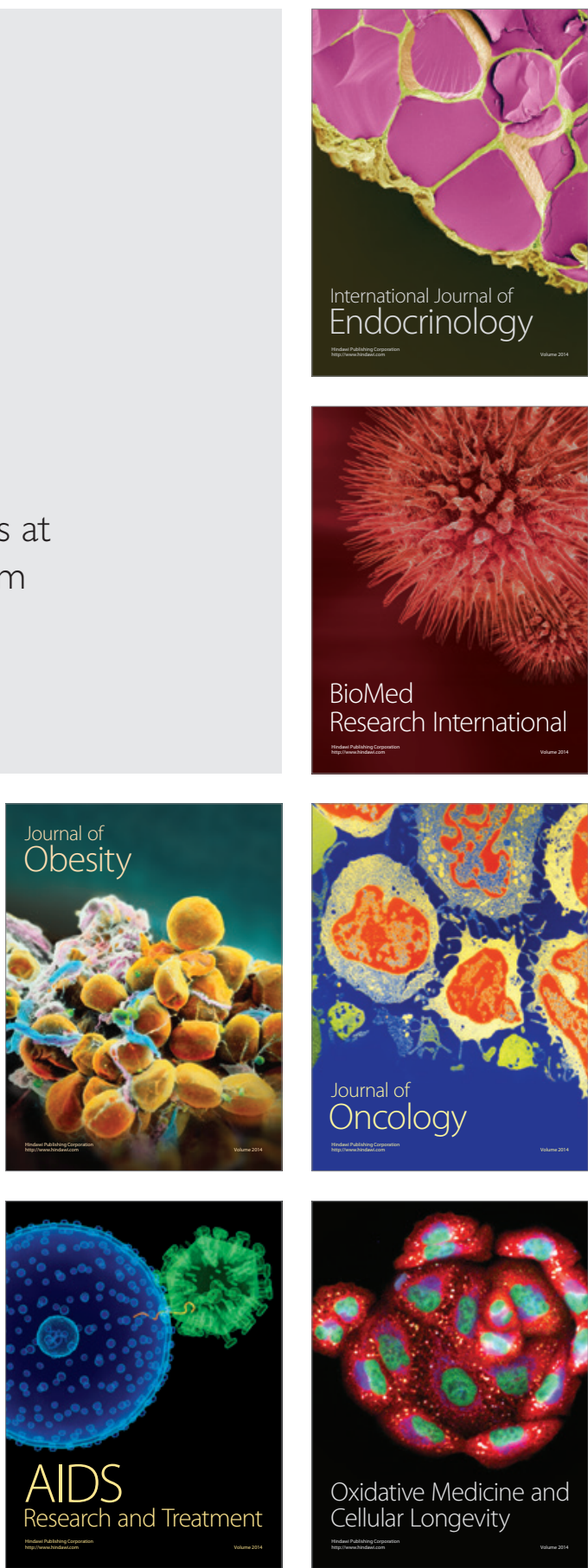